\title{
A Novel Multi-Modal Platform To Image Molecular And Elemental Alterations In
}

\section{Ischemic Stroke}

Sally Caine ${ }^{* A}$, Mark J Hackett ${ }^{*}$, Huishu Hou ${ }^{\mathrm{A}}$, Saroj Kumar ${ }^{\mathrm{C}}$, Jason Maley ${ }^{\mathrm{D}}$, Zurab Ivanishvili ${ }^{\mathrm{A}}$, Brandon Suen $^{\mathrm{A}}$, Aleksander Szmigielski ${ }^{\mathrm{A}}$, Zhongxiang Jiang ${ }^{\mathrm{E}}$, Nicole J Sylvain ${ }^{\mathrm{A}}$, Helen

Nichol $^{\mathrm{A}}$, Michael E Kelly ${ }^{* * A, F}$

*Denotes shared first authorship

** Corresponding Author Email: michael.kelly@usask.ca $\quad$ Phone: 1-306-844-1104

${ }^{A}$ Department of Anatomy and Cell Biology, University of Saskatchewan, 107 Wiggins Road, Saskatoon, Saskatchewan S7N 5E5, Canada

${ }^{B}$ Molecular and Environmental Sciences Group, Geological Sciences, University of Saskatchewan, 114 Science Place,

Saskatoon, Saskatchewan S7N5E2, Canada

${ }^{C}$ Canadian Light Source, SK, Canada

${ }^{D}$ Saskatchewan Structural Sciences Center, University of Saskatchewan, 110 Science Place, Saskatoon, SK S7N 5C9, Canada

${ }^{E}$ Leica Corporate, Germany

${ }^{F}$ Division of Neurosurgery, Department of Surgery, College of Medicine, University of Saskatchewan, Royal University Hospital, 103 Hospital Drive, Saskatoon, SK S7N 0W8, Canada

\section{Key Words}

Biospectroscopy, Stroke, Ischemia, Edema, Photothrombotic, Neurodegeneration

\section{Abbreviations}

SR-FTIR Synchrotron Radiation Fourier Transform Infrared

IAUC Integrated Area Under the Curve

PIZ Peri-Infarct Zone

CARS Coherent Anti-Stokes Raman Spectroscopy 


\begin{abstract}
Stroke is a major global health problem, with the prevalence and economic burden predicted to increase due to aging populations in western society. Following stroke, numerous biochemical alterations occur and damage can spread to nearby tissue. This zone of "at risk" tissue is termed the peri-infarct zone (PIZ). As the PIZ contains tissue not initially damaged by the stroke, it is considered by many as salvageable tissue. For this reason, much research effort has been undertaken to improve the identification of the PIZ and to elucidate the biochemical mechanisms that drive tissue damage in the PIZ in the hope of identify new therapeutic targets. Despite this effort, few therapies have evolved, attributed in part, to an incomplete understanding of the biochemical mechanisms driving tissue damage in the PIZ. Magnetic resonance imaging (MRI) has long been the gold standard to study alterations in gross brain structure, and is frequently used to study the PIZ following stroke. Unfortunately, MRI does not have sufficient spatial resolution to study individual cells within the brain, and reveals little information on the biochemical mechanisms driving tissue damage. MRI results may be complemented with histology or immuno-histochemistry to provide information at the cellular or sub-cellular level, but are limited to studying biochemical markers that can be successfully "tagged" with a stain or antigen. However, many important biochemical markers cannot be studied with traditional MRI or histology/histochemical methods. Therefore, we have developed and applied a multi-modal imaging platform to reveal elemental and molecular alterations that could not previously be imaged by other traditional methods. Our imaging platform incorporates a suite of spectroscopic imaging techniques; Fourier transform infrared imaging, Raman spectroscopic imaging, Coherent anti-stoke Raman spectroscopic imaging and X-ray fluorescence imaging. This approach does not preclude the use of traditional imaging techniques, and rather it should be use to complement traditional methods such as MRI or histology and immunohistochemistry, to gain a greater insight into disease mechanisms. We demonstrate the potential of this approach by characterizing biochemical alterations within the PIZ 24 hours after the induction of photothrombotic stroke in mice. Substantial molecular and elemental alterations were identified in the PIZ 24 hours after stroke that are consistent with tissue swelling and edema, but not oxidative stress. This reveals important mechanistic information, that could not previously be obtained, which should be considered in future studies aimed at developing therapeutic intervention from this model.
\end{abstract}




\subsection{Introduction}

Stroke is a major global cause of adult morbidity and mortality, and the associated hospitalization and physician services required for stroke victims, alongside lost wages and decreased productivity of the individual is a huge international economic concern. ${ }^{1}$ The development of innovative and restorative therapies are needed to reduce the tremendous burden that stroke has on society. However, it is likely that these treatments will only become known by obtaining a better understanding of the biochemical and cellular mechanisms of stroke.

Brain injury associated with ischemic stroke results from a reduction in blood flow causing a decrease in both oxygen and nutrients to the affected region of the brain, ultimately resulting in cell death. ${ }^{2}$ The high metabolic demands of neurons make them particularly vulnerable to ischemic insult. In addition, cells killed or irreversibly damaged by the ischemic insult can release factors, including water (i.e., edema), which may spread into and harm nearby tissue that was not initially damaged by the stroke. ${ }^{2-5}$ This process can be observed as delayed or ongoing cell death following the ischemic insult. ${ }^{2,3,5}$ Tissue regions that surround the ischemic infarct, which are at risk of ongoing tissue damage are often termed the peri-infarct zone (PIZ). ${ }^{6,7}$ In addition to the spread of toxic products from the ischemic infarct into the PIZ, glial and macrophage activation from the ensuing inflammatory response often occur in this region and may also contribute to ongoing cell death ${ }^{3,7-12}$. The PIZ is also a major site of brain plasticity associated with spontaneous recovery and compensation after stroke. ${ }^{13,14}$ Taken together, the delayed and ongoing tissue damage within the PIZ may increase the size of the ischemic infarct and hamper repair and recovery, contribute to worsening disability. Thus, further elucidation of the biochemical mechanisms underpinning delayed and ongoing cell death within the PIZ are required to aid in identifying novel therapeutic interventions.

The photothrombotic model of focal ischemia, in which a blood clot is formed by fluorescence excitation of a photosensitive dye, rose bengal, is commonly used in rodents to study stroke. This animal model has been highly beneficial for investigating neuroplasticity and remodelling of brain tissue following ischemic damage, and has the advantage of a minimally invasive surgery and the ability to precisely control the size and anatomical location of the infarct within the cerebral cortex. ${ }^{6}$ However, the ischemic lesion created in this model results from complete cessation of blood flow and is thought to contain little or no oxidative damage associated with free radical production during reperfusion. ${ }^{6,7,15}$ Other stroke models, such as 
middle cerebral artery occlusion (MCAO), or stroke patients usually have a significant ischemic penumbra (i.e., a tissue region with reduced blood flow not sufficient to cause immediate energy failure and cell death). ${ }^{16-18}$ In contrast, the photothrombotic model is thought to have minimal or no ischemic penumbra and based on the extent of reperfusion and edema may more accurately reflect the pathophysiology associated with traumatic brain injury. ${ }^{6}$ Thus, further characterization of the altered neurochemistry associated with the photothrombotic stroke model is required to validate the use of this model to study the biochemical mechanisms responsible for cell death following ischemic insult.

Previous studies have characterized the anatomical and biochemical alterations occurring in the photothrombotic stroke model using conventional techniques, such as routine histology, $4,19,20$ immunohistochemistry, ${ }^{4}$ magnetic resonance imaging (MRI), ${ }^{4,20}$ or auto-radiography. ${ }^{21}$ Despite the valuable information garnered with these methods, they suffer from a lack of spatial resolution or provide only a limited view of the biochemical "picture". For example, MRI and auto-radiography have a spatial resolution on the order of hundreds of microns, which is not sufficient to study alterations at the cellular level. Furthermore, although MRI is well suited to study gross anatomical alterations to brain tissue, it does not reveal a wealth of biochemical information. Conversely, histology and immunohistochemistry provide high spatial resolution images but are limited to biochemical markers that can be successfully localized with an antibody or stain. On the other hand, tissue micro-dissection and biochemical assay can assess a wealth of biochemical parameters, but important anatomical information is lost. An advantage of magnetic resonance is that it can be used to detect a range of biochemical parameters (i.e., lactate and organic phosphates) in vivo in living animals, allowing time course or functional studies of brain biochemistry. However, the spatial resolution of the information revealed is similar to that obtained through tissue micro-dissection and biochemical assay. Recently, spectroscopic techniques that provide direct in situ elemental or molecular imaging at near cellular, cellular or sub-cellular spatial resolution have been applied in the field of neuroscience. These techniques include Fourier transform infrared (FTIR) spectroscopic imaging, Raman spectroscopy, Coherent anti-Stokes Raman spectroscopy (CARS) and X-ray fluorescence imaging (XFI). These techniques provide the unique possibility to image at cellular resolution, the distribution of biochemical markers that could previously only be studied in bulk with biochemical assay. Furthermore, both spectroscopic imaging and histology can be performed on the same tissue 
section or serial tissue sections, allowing direct correlation between cell morphology and the cellular distribution of biochemical markers.

FTIRI has been previously used to image molecular alterations associated with neurodegeneration in animal models of Alzheimer's disease, ${ }^{22-26}$ Parkinson's disease, ${ }^{27}$ amyotrophic lateral sclerosis, ${ }^{28}$ multiple sclerosis, ${ }^{29}$ cerebral malaria, ${ }^{30}$ epilepsy ${ }^{31,32}$ and hemorrhagic stroke. ${ }^{33}$ FTIR imaging is well suited to image important biochemical markers associated with neurodegeneration, such as total lipid, total protein levels and alterations in protein secondary structure (i.e., increased concentration of protein aggregates). ${ }^{34-36}$ Although imaging is typically performed with benchtop instruments and thermal sources, at a spatial resolution of $10-20 \mu \mathrm{m}$, the recent coupling of synchrotron infrared light sources with focal plane array detectors provide sub-micron pixel resolution and the ability to perform sub-cellular molecular imaging. ${ }^{36-42}$ Similar to FTIR imaging, Raman spectroscopy is sensitive to molecular vibrations, and has found extensive use to quantify and image lipids in biological samples. ${ }^{43-46} \mathrm{~A}$ weaker signal is obtained with Raman scattering relative to FTIR imaging (i.e., Raman has poorer detection limits), however, the shorter wavelengths of light used in Raman spectroscopy/microscopy allow higher spatial resolution imaging. Further, Coherent anti-Stokes Raman scattering (CARS) alleviates background fluorescence thus enabling rapid sub-micron lipid imaging. ${ }^{47}$ The biomolecular information provided by FTIR imaging, Raman spectroscopy and CARS can be complemented by techniques such as XFI which provide a wealth of elemental information. XFI has been previously used to study the distribution of transition metals ( $\mathrm{Fe}, \mathrm{Cu}$, $\mathrm{Zn})$ and ions $\left(\mathrm{Cl}^{-}, \mathrm{K}^{+}\right.$, and $\left.\mathrm{Ca}^{++}\right)$following brain degeneration associated with Alzheimer's disease ${ }^{48,49}$ Parkinson's disease, ${ }^{50,51}$ amyotrophic lateral sclerosis, ${ }^{52}$ haemorrhagic stroke ${ }^{33}$ as well as following epileptic seizures, ${ }^{53-55}$ and traumatic brain injury. ${ }^{56}$ Together, these techniques yield a plethora of biochemical information that can be coupled with traditional histology or immunohistochemistry methods. Whilst, several studies have combined one or two of the above spectroscopic imaging methods in the same study, ${ }^{23,57}$ thus far, none have employed the full suite of techniques. In this study, we combined FTIR imaging, Raman spectroscopy, CARS microscopy and XFI with routine histological/immunohistochemistry of serial brain tissue sections to characterize the neurochemical changes that occur within the cortex following photothrombotic stroke in mice. Our results provide new insight into the biochemical alterations that occur within this animal model, particularly within the PIZ, and lay the foundation for future 
time-course studies of stroke mechanisms. Furthermore, this study demonstrates the immense research potential associated with the application of this novel suite of imaging techniques, especially when used in combination with traditional methods, such as histology and immunohistochemistry.

\subsection{Materials and Methods}

\subsection{Animal model}

Male Balb/c mice, aged 11 weeks, were housed with a $12 \mathrm{~h}$ light/dark cycle with access to chow and water ad libitum. This work was approved by the University of Saskatchewan's Animal Research Ethics Board, and adhered to the Canadian Council on Animal Care guidelines

for humane animal use. Photothrombotic stroke was induced as previously described. ${ }^{58}$ Briefly, mice $(n=6)$ were anesthetized using isoflurane (2\% induction, $1.5-2 \%$ maintenance in $40 \% \mathrm{O}_{2}$ and $60 \% \mathrm{~N}_{2} \mathrm{O}$, Baxter Corporation, Toronto ON, CA). The fur above the skull was removed using a handheld shaver (Braun Cruzer, Proctor \& Gamble, CA) and the head was secured in a stereotaxic frame. Throughout the procedure, body temperature was maintained using a RightTemp® temperature monitor and homeothermic controller (Kent Scientific Corporation, USA), and breathing rate, pulse and oxygen saturation was monitored using a MouseOX Plus (Starr Life Sciences Corp., USA). To expose the skull, a midline incision of the scalp was made. Using the stereotaxic apparatus, we identified the area of the skull overlying the primary somatosensory cortex (S1FL), according to published coordinates ( -1 to $-2.5 \mathrm{~mm}$ midline to lateral; +1 to -0.5 anterior to posterior of the bregma, right hemisphere). The mice were given an intraperitoneal injection of Rose Bengal (100mg/kg body weight, Sigma, MO, USA), followed by a 5 min. wait period to ensure the dye had time to circulate through the animal. A sterile mask was placed on the surrounding area of the region of interest to reduce scatter of the laser light, and the S1FL was illuminated for 20 mins with a green laser $(532 \mathrm{~nm}$, located $3 \mathrm{~cm}$ above the skull) to photoactivate the rose bengal. For sham mice $(n=3)$, we administered the dye and performed all other procedures, including the 20 min illumination period, but omitted exposure to the laser. Marcaine $(1.67 \mathrm{mg} / \mathrm{ml}, 18 \mathrm{ul} / 15 \mathrm{~g}$ body weight) was applied to the open wound prior to suturing the incision. After surgery, animals were allowed to recover in single cages until they were sacrificed 24 hours after the induction of stroke (e.g. when the laser was 
turned off). Routine histology was performed on sham operated mice to confirm normal tissue morphology with no ischemic infarct (Supporting Information Figure 2). Thus, all further comparisons were made only between the ipsilateral hemisphere and contralateral hemisphere of ischemic mice.

\subsection{Sample preparation}

To avoid introduction of chemical artifacts that can result during sample preparation of biological samples, ${ }^{30,39,59,60}$ all mice were anaesthetized with isoflurane, and sacrificed through decapitation, with the head immediately frozen by dropping into liquid nitrogen, as described

previously. ${ }^{61}$ The frozen brain was chiseled out from the head on dry ice. For each brain, ten 10$\mu$ m-thick coronal section of the brain were cut with a cryo-microtome at $-18{ }^{\circ} \mathrm{C}$ from the central location of the lesion ( $+0.25 \mathrm{~mm}$ anterior to Bregma). Of these, one section was melted onto a $\mathrm{CaF}_{2}$ substrate for FTIR imaging analysis, one section was melted onto a metal-free plastic Thermanox TM coverslip for XFI analysis and eight sections were mounted onto regular glass microscope slides. From the sections mounted onto glass microscope slides one section was used for Raman spectroscopic imaging, one section was used for CARS imaging, and the remaining six sections were used for histology and immunohistochemical analyses. Following tissue sectioning and mounting on the respective substrate, each tissue sample was stored at $-80{ }^{\circ} \mathrm{C}$ until analyzed. Directly prior to analysis, each tissue section was immediately air-dried at ambient laboratory temperature in a desiccator. The tissue sections on microscope glass slides were post-fixed by immersion with $10 \%$ buffered formalin prior to staining.

\subsection{Globar FTIR imaging}

Globar-FTIR spectroscopic imaging was performed at the Canadian Light Source (CLS) with a Hyperion 3000 microscope fitted with an upper objective of $15 \times$ magnification and a numerical aperture of 0.4 , and a lower condenser of $15 \times$ magnification and 0.4 numerical aperture. This arrangement yielded a pixel size of $2.65 \times 2.65 \mu \mathrm{m}$, which was later subjected to $8 \times 8$ pixel binning to yield an effective image pixel size of $21.2 \times 21.2 \mu \mathrm{m}$. Globar-FTIR images were acquired with a spectral resolution of $4 \mathrm{~cm}^{-1}$ with the co-addition of 16 scans. A background image was collected from blank substrate using 16 co-added scans immediately prior to each sample. 


\subsection{Single-beam wide-field SR-FTIR imaging}

For each animal, SR-FTIR images were collected from 3 cortical pyramidal neurons within the PIZ and from three cortical pyramidal neurons at the corresponding location in the adjacent hemisphere (contralateral hemisphere). Single-beam wide-field SR-FTIR imaging was performed at the Canadian Light Source (CLS) with a Hyperion 3000 microscope fitted with an upper objective of $52 \times$ magnification and a N.A. of 0.65 , and with a lower condenser of $32 \times$ magnification and a N.A. of 0.65 . This arrangement yielded $0.77 \times 0.77 \mu \mathrm{m}$ pixel size. The infrared beam was focused and aligned to the center of the array, then defocused such that an array area of approximately $25 \times 25 \mu \mathrm{m}(32 \times 32$ pixels $)$ was quasi-homogeneously illuminated. This approach was identical to that previously reported to image pyramidal neurons of the cortex using single-beam wide-field SR-FTIR imaging. ${ }^{42}$

Spectra were collected with the co-addition of 512 scans, at $4 \mathrm{~cm}^{-1}$ resolution. A background spectrum (512 co-added scans) was collected from blank substrate before each sample measurement, to minimize the effect of beam current decay on data. As previously described, normalization to beam current was not performed. ${ }^{39}$ During data processing individual images were cropped to the region of interest surrounding individual neurons.

\subsection{Data processing and data analysis of FTIR spectra}

All data processing and image generation was performed using Cytospec software (Cytospec, Version 1.2.04) and Opus software (Version 6.5, Bruker, Ettlingen, Germany). False colour functional group images of lipid acyl groups, lipid esters and total protein were generated from the integrated area underneath the curve (IAUC) in raw spectra. Areas were integrated across the range $2865-2845 \mathrm{~cm}^{-1}, 1755-1715 \mathrm{~cm}^{-1}$ and $1700-1600 \mathrm{~cm}^{-1}$ for lipid acyl groups $v_{\mathrm{s}}\left(\mathrm{CH}_{2}\right)$, lipid ester $v(\mathrm{C}=\mathrm{O})$, and protein amide I band, respectively. A linear baseline was subtracted across the same range as integration. To calculate the average spectrum of each individual neuron, a region of interest was drawn to encompass the neuron soma which was guided by an overlay of bright field microscopy images, as indicated in Supporting Information Figure 1.

\subsection{Raman Spectroscopic Imaging Data Collection}


Raman spectroscopy measurements were carried out on a Renishaw InVia Reflex Raman microscope using an $\mathrm{Ar}^{+}$laser (Stellar Pro-50, Modu-Laser LLC, Centerville, UT) operating at $514.5 \mathrm{~nm}$, and an 1800 lines/mm grating. The microscope was focused onto the sample using a Leica 100X (NA = 0.90) objective, and the backscattered Raman signals were collected with a Peltier cooled CCD detector. High resolution Raman imaging $(0.6 \mu \mathrm{m}$ x $0.6 \mu \mathrm{m}$ pixel size $)$ was acquired using Streamline ${ }^{\mathrm{TM}}$ mode within the instrument software (Renishaw Wire ${ }^{\mathrm{TM}}$ V3.4) using a 30s exposure time and a static scan centered at $2900 \mathrm{~cm}^{-1}$ (the combination of laser excitation and grating gives an effective spectrum range of $\left.2710-3200 \mathrm{~cm}^{-1}\right)$. Tissue samples (5$10 \mu \mathrm{m}$ thickness) were fixed on an Au-coated Si wafer, and sample imaging was completed within $72 \mathrm{hr}$. of tissue section preparation. The $514.5 \mathrm{~nm}$ laser power was $1.5 \mathrm{~mW}$ measured at the sample. The instrument's calibration was verified using an internal Si (110) sample, which was measured at $520 \mathrm{~cm}^{-1}$.

\subsection{Raman Spectroscopic Data Processing}

Raman image processing was performed using Renishaw Wire (V3.4) and Gwyddion free-ware image processing software (V2.40). Images were processed using the cosmic ray removal and noise filtering function within the instrument software program (Rensishaw Wire ${ }^{\mathrm{TM}}$ V3.4). Lipid acyl images were generated from the 2830-60 $\mathrm{cm}^{-1}$ integrated area for the $v_{\mathrm{s}}\left(\mathrm{CH}_{2}\right)$ vibrational band. Image display and average values collected for statistical analysis were generated with Gwyddion software by drawing the region of interest around the neuron soma was guided by an overlay of bright field microscopy images, similar to that performed for FTIR imaging and as indicated in Supporting Information Figure 1.

\subsection{CARS Data Collection}

CARS imaging was performed with a Leica TCS SP8 CARS system (Leica Microsystems, Mannheim, Germany) based on a TCS SP8 confocal microscope. A near infrared laser picoEmerald (APE, Berlin, Germany) offering a fixed Stokes laser line of $1064.5 \mathrm{~nm}$ and a tuneable Pump line from an optical parametric oscillator $(780-940 \mathrm{~nm})$ was used to generate CARS signal. Raman vibration of $1250-3400 \mathrm{~cm}^{-1}$ can be imaged with the system. Either a 40x HC PL IRAPO Corr water immersion or a 20× 0.75 HC PL APO Imm Corr CS2 objective was used for the imaging and CARS signal was detected with a non-descanned photon multiplier tube 
detector at the reflected light side. The CARS signal was selected with a CARS2000 filter cube $\left(2000-3400 \mathrm{~cm}^{-1}\right)$ placed in front of the detector. For imaging of $\mathrm{CH}_{2}$ vibration with Raman shift of $2863 \mathrm{~cm}^{-1}$ pump wavelength of $815.8 \mathrm{~nm}$ was used. Spectral scan was performed with the integrated $x y \Lambda$ scan mode automatically after setting the range to be scanned either by Raman wave numbers or by pump wave lengths as needed.

\subsection{XFI data collection}

Large scale elemental maps of the coronal brain tissue sections were collected using XFI at the micron level at the Stanford Synchrotron Radiation Lightsource (SSRL) experimental station 10-2 (http://www-ssrl.slac.stanford.edu/beamlines/bl10-2/). The energy of the incident Xray beam was $13.45 \mathrm{keV}$. Incident X-ray intensity was measured using a nitrogen gas-filled ion chamber. A $50 \mu \mathrm{m}$ pin-hole aperture was used to define the beam size on the sample. The sample was mounted at $45^{\circ}$ to the incident beam and raster scanned through the beam ("fly" scan or "rapid" scan mode), with a beam exposure time of $200 \mathrm{~ms}$ per $30 \mu \mathrm{m}$ step. A single-element Vortex ${ }^{\circledR}$ silicon drift detector at $90^{\circ}$ to the incident beam collected the X-ray fluorescence spectra from the brain tissue. The full emission spectrum via multichannel analysis (MCA) was recorded at each pixel, in addition to single channels for $\mathrm{K}, \mathrm{Ca}, \mathrm{Fe}, \mathrm{Cu}$, and $\mathrm{Zn}$ fluorescence lines, and elastic scatter. Elements below the Ar K-edge were not recorded (P, S, Cl). Emission spectra were also collected from a series of standard foils of known elemental concentration to quantify the single channel data: $\mathrm{K}\left(\mathrm{KCl}, 98.8 \mu \mathrm{g} \mathrm{cm}^{-2}\right)$; $\mathrm{Ca}\left(\mathrm{CaF}_{2}, 56.8 \mu \mathrm{g} \mathrm{cm}^{-2}\right)$; Fe $(\mathrm{Fe}, 56.0 \mu \mathrm{g}$ $\left.\mathrm{cm}^{-2}\right)$; $\mathrm{Cu}\left(\mathrm{CuS}, 49.7 \mu \mathrm{g} \mathrm{cm}^{-2}\right)$; and $\mathrm{Zn}\left(\mathrm{ZnTe}, 45.8 \mu \mathrm{g} \mathrm{cm}^{-2}\right)$ (Micromatter, Vancouver).

\subsection{XFI data processing}

XFI data was viewed and analyzed using Sam's Microanalysis Toolkit (http://smak.samsxrays.com/). The XFI elemental maps were converted from fluorescence intensity to elemental concentration $\left(\mu \mathrm{g} / \mathrm{cm}^{2}\right)$, using reference standards, as previously described. ${ }^{62}$ Regions of interest were defined to correspond to the ischemic infarct, PIZ and contralateral hemisphere and the average elemental concentration calculated within each region.

\subsection{Histology/immunohistochemistry}


For histological analysis, slides were fixed in $10 \%$ buffered formalin and stained with hematoxylin and eosin (H\&E). Lactase de-hydrogenase (LDH, Abcam, ab52488, Cambridge, USA) immunohistochemistry was performed on adjacent slides using a protocol obtained from Biolegend. Following fixation, slides were rinsed in phosphate buffered saline (PBS, 10mM), blocked in a $0.3 \%$ peroxide solution in PBS for 10 mins, rinsed in PBS, then blocked for $1 \mathrm{~h}$ with $10 \%$ fetal bovine serum in PBS, following by rinsing in PBS. Slides were incubated for $1 \mathrm{~h}$ in anti-LDH antibody at 1:50 in 0.5\% bovine serum albumin in PBS, rinsed in PBS, incubated for 30 mins in pre-diluted streptavidin-HRP, rinsed again in PBS, and incubated for 10 mins fresh $\mathrm{DAB}$ solution $(0.05 \% \mathrm{DAB}, 0.015 \%$ peroxide, $\mathrm{PBS})$. To finish, the slides were rinsed, dehydrated in ethanol and then cleared in Xylene before coverslipping. For anti-Glial Fibrillary Acidic Protein (GFAP, Dako, Z0334, Glostrup, Denmark) immunohistochemistry, adjacent slides were immuno-labelled using a Dako EnVision Plus reagents kit (Dako, Denmark), with the aid of an hydration chamber to prevent dehydration. Briefly, frozen sections air dried onto slides were fixed with 10\% methanol free formalin (prepared freshly from paraformaldehyde) for 5 mins and then rinsed with PBS with $0.1 \%$ Tween 20 (PBST). The slides were then blocked with Dako Peroxidase Block for 10 mins, rinsed with PBST, and incubated for 30 mins in GFAP diluted at 1:1500 in Dako Antibody diluent. After being rinsed in PBST, the slides were incubated for 30 mins in Dako Labelled polymer (HRP anti-rabbit), then rinsed in PBST and incubated in Dako DAB substrate buffer mixed freshly with Dako DAB Chromagen for 10 mins. To finish, the slides were incubated for 5 mins in fresh $2 \%$ copper sulfate, then rinsed in distilled water, dehydrated in ethanol, cleared in xylene and coverslipped. All procedures were done at room temperature unless otherwise stated.

\subsection{Statistical Analysis}

A paired $t$-test was used to assess significant differences between the elemental or molecular content of the ischemic infarct and the PIZ; the ischemic infarct and the healthy contralateral tissue; and between the PIZ and the contralateral tissue. As multiple $t$-test comparisons were used, the $p$-value obtained was multiplied by the number of $t$-tests performed (3) to control for familywise error. For high resolution SR-FTIR imaging and Raman data, the molecular content of the neuron soma was compared to the neuropil with a paired $t$-test. All $t$ tests were two tailed and the $95 \%$ confidence limit was used to test for significance. 


\subsection{Results}

\subsection{Histopathology: Evidence of edema and gliosis in the PIZ}

The ischemic infarct shows an absence of eosin staining of the neuropil within the ischemic infarct (Figure 1A). Tissue immediately surrounding the ischemic infarct shows patchy eosin staining that is paler than tissue at a greater distance from the infarct (Figure 1D). Pale and patchy eosin staining within the PIZ is a characteristic feature of edema. Cells within the ischemic infarct typically have shrunken, pyknotic nuclei and an eosinophilic cytoplasm, a histological marker of degenerating cells after ischemic insult (black arrow, Figure 1D). Similarly, LDH immunohistochemistry, a marker of cell integrity revealed little or no staining within the ischemic infarct (Figure 1B). However, some cells within the ischemic infarct showed intense LDH antigenicity at the periphery of the cell but little or no staining within the center of the cell (black arrows, Figure 1E). This is in contrast to cells in the PIZ or contralateral hemisphere, which displayed strong staining throughout the cell body. The anatomical boundary between healthy tissue and the PIZ could not be distinguished based on the LDH distribution. Immunohistochemical localization of GFAP was used to detect gliosis following ischemic insult. Substantial GFAP staining of cells with morphology consistent with glia, was observed surrounding the infarct consistent with the location of the PIZ (Figure 1C, F). No GFAP antigenicity was observed within the ischemic infarct.

\subsection{Globar-FTIR imaging: Lipid and protein content is reduced in the PIZ relative to the} contralateral hemisphere.

FTIR imaging with a thermal globar source was used to survey large regions of tissue to identify biochemical alterations within the ischemic core and PIZ relative to the contralateral hemisphere. Representative images of the distribution of lipid acyl groups, lipid esters, total protein and aggregated protein content alongside routine H\&E histology are presented in Figure 2A. The relative distribution of lipid acyl groups, lipid esters and total protein were generated from the IAUC of the $v_{\mathrm{s}}\left(\mathrm{CH}_{2}\right)$ band $\left(2865-2845 \mathrm{~cm}^{-1}\right), v(\mathrm{C}=\mathrm{O})$ band $\left(1755-1715 \mathrm{~cm}^{-1}\right)$ and amide I band $\left(1700-1600 \mathrm{~cm}^{-1}\right)$, respectively. ${ }^{39,40,42}$ The relative distribution of aggregated protein was determined from second-derivative intensity at $1625 \mathrm{~cm}^{-1} .33$ The level of relative aggregated protein content was quantified from second-derivative intensity and from curve 
fitting the original spectra, as previously described. ${ }^{33}$ Lipid acyl groups, lipid esters and total protein levels decreased within the infarct, while aggregated protein increased (Figure 2B). The images reveal a subtle decrease in lipid acyl groups, lipid esters and total protein within the PIZ, whilst protein aggregates did not increase within this region (Figure 2C). These visual observations are reflected in the average spectra of each tissue region (infarct, PIZ, contralateral hemisphere), as shown in Figure 2C. Analysis of these changes across 6 animal replicates indicated that the average lipid acyl group content was significantly reduced in the infarct $(0.16 \pm$ $0.042 \mathrm{AU})$ when compared to the PIZ $(0.33 \pm 0.45 \mathrm{AU})$ and both were significantly reduced when compared with the contralateral hemisphere $(0.44 \pm 0.026 \mathrm{AU})$. A similar pattern was observed for the lipid ester and total protein content, with the infarct having significantly lower levels $(0.22 \pm 0.10 \mathrm{AU}$ and $6.0 \pm 1.6 \mathrm{AU})$ when compared with the PIZ $(0.42 \pm 0.092 \mathrm{AU}$ and 11 $\pm 1.5 \mathrm{AU})$ and both regions were significantly reduced in comparison to the contralateral hemisphere $(0.54 \pm 0.066 \mathrm{AU}, 14 \pm 1.2 \mathrm{AU})$, respectively (Figure 2D). In contrast, the infarct had significantly increased aggregated protein content in comparison with the PIZ and contralateral hemisphere, which were not significantly different from one another (Figure D). Representative examples of second-derivative spectra show an increase in intensity at $1625 \mathrm{~cm}^{-1}$ consistent with increased relative aggregated protein content within the ischemic infarct relative to the PIZ and contralateral hemisphere (Figure 2E). The increase in second-derivative intensity at $1625 \mathrm{~cm}^{-1}$ corresponds with decreased intensity at $1656 \mathrm{~cm}^{-1}$, which is attributed to a loss of protein with an $\alpha$-helical secondary structure. A representative example of the curve fitting procedure used to quantify the relative aggregated protein content is shown in Figure 2E. Interestingly, despite the large decrease in total lipid and total protein content within the PIZ, the lipid to protein ratio within the PIZ was similar to the healthy appearing tissue in the contralateral hemisphere. The lipid ester to protein ratio was $0.038 \pm 0.002 \mathrm{AU}$ and $0.038 \pm$ $0.004 \mathrm{AU}$ for the contralateral hemisphere and PIZ, respectively ( $p$ value $=0.74$ ).

3.03 Wide-field SR-FTIR imaging: The soma of neurons in the PIZ have decreased protein content and the neuropil has decreased lipid content

Wide-field SR-FTIR imaging is a recent imaging advancement that provides rapid direct ex vivo biochemical imaging in situ within tissue sections at a spatial resolution of $2-5 \mu$. $^{41}$ Localization of individual cells and larger sub-cellular features (i.e., nucleus) has been achieved 
using both multi-beam wide-field imaging, ${ }^{34,36,37,39}$ and single-beam wide-field imaging. ${ }^{36,42}$ In this study, single-beam wide-field SR-FTIR imaging was used for direct biochemical imaging of the lipid and protein content within the soma of individual neurons and the surrounding neuropil within the PIZ and in the contralateral hemisphere. Representative examples of the relative distribution of lipid acyl groups, lipid esters, and total protein are presented in Figure 3A. Together, the images and representative spectra highlight that there is a decrease in the relative content of lipid acyl groups, lipid ester groups and total protein within the neuropil of the PIZ of the ipsilateral hemisphere relative to the contralateral hemisphere (Figure 3A, B). Quantification of the bands confirmed that these visual observations were statistically significant, with lipid acyl groups reducing from $0.62 \pm 0.078 \mathrm{AU}$ to $0.49 \pm 0.085 \mathrm{AU}$; lipid ester groups from $0.79 \pm 0.13$ AU to $0.56 \pm 0.032 \mathrm{AU}$; and total protein from $18 \pm 3.9 \mathrm{AU}$ to $12 \pm 0.51 \mathrm{AU}$, in the neuropil in the PIZ in contralateral and ipsilateral hemispheres, respectively. In contrast, the content of lipid acyl groups $(0.35 \pm 0.075 \mathrm{AU}$ and $0.38 \pm 0.067 \mathrm{AU})$ and lipid esters $(0.32 \pm 0.074 \mathrm{AU}$ and 0.42 $\pm 0.32 \mathrm{AU})$ in the soma of neurons were not significantly different between the ipsilateral and contralateral hemispheres, respectively. However, total protein content within soma of neurons in the PIZ $(13 \pm 0.92)$ compared to the contralateral hemisphere $(18 \pm 3.1)$ was significantly decreased.

3.04 Raman spectroscopic imaging validates wide-field SR-FTIR imaging and demonstrates reduced lipid content within the neuropil inside the PIZ

To corroborate the results of wide-field SR-FTIR imaging, Raman spectroscopic imaging was used to image the distribution of lipid acyl groups at high spatial resolution $0.5 \mu \mathrm{m}$. The relative distribution of lipid acyl groups was generated from the IAUC of the $v_{\mathrm{s}}\left(\mathrm{CH}_{2}\right)$ band (2865 $-2845 \mathrm{~cm}^{-1}$ ). The representative images (Figure 4A) and spectra (Figure 4B) show a decrease in the relative content of lipid acyl groups within the neuropil of the PIZ of the ipsilateral hemisphere relative to the contralateral hemisphere, and are in strong agreement with the finding obtained from wide-field SR-FTIR imaging. Quantification of the lipid acyl group was significantly reduced in the neuropil in the PIZ of the ipsilateral hemisphere compared with the contralateral hemisphere (Figure 4C).

3.05 CARS: Loss of lipid is associated with loss of myelin within the PIZ 
CARS imaging was used to reveal the distribution of lipids at sub-organelle spatial resolution, as described by others. ${ }^{47,63-65}$ As shown in Figure 5A-B, the distribution of lipids imaged with CARS $v_{\mathrm{s}}\left(\mathrm{CH}_{2}\right)$ shows the characteristic appearance of myelinated axons (i.e., two lipid rich margins bordering the axon). Numerous myelinated axons can be observed in the cerebral cortex of the contralateral hemisphere, however, myelinated axons are largely absent in the ischemic infarct and PIZ (Figure 5C, D).

\subsection{XFI: reveals disturbed elemental homeostasis within the PIZ}

XFI with a $50 \mu \mathrm{m}$ pin-hole aperture was used to survey large regions of tissue with a 30 $\mu \mathrm{m}$ step size to identify elemental alterations within the ischemic infarct and PIZ relative to the contralateral hemisphere. Representative images of the distribution of elements $(\mathrm{K}, \mathrm{Ca}, \mathrm{Fe}, \mathrm{Cu}$, $\mathrm{Zn}$ ) alongside routine $\mathrm{H} \& \mathrm{E}$ histology are presented in Figure 6A. The average elemental concentration was quantified for tissue regions within the ischemic infarct and PIZ, as well as within the equivalent cortical region of the contralateral hemisphere (Figure 6B). The concentration of all elements $(\mathrm{K}, \mathrm{Ca}, \mathrm{Fe}, \mathrm{Cu}, \mathrm{Zn})$ decreased significantly within the infarct relative to the PIZ and the contralateral hemisphere (Figure 6B). The concentration of all elements $(\mathrm{K}, \mathrm{Ca}, \mathrm{Fe}, \mathrm{Cu}, \mathrm{Zn})$ was decreased within the PIZ relative to the contralateral hemisphere, however, only the decrease in $\mathrm{K}$ and $\mathrm{Ca}$ were statistically significant.

\subsection{Discussion}

4.01 FTIR imaging and XFI complement routine histology and immunohistochemistry and provide clear delineation of the PIZ

We have applied a novel suite of direct in situ biospectroscopic imaging techniques to study the molecular and elemental alterations that occur within the PIZ following ischemic insult. Routine histology and LDH immunohistochemistry provided clear identification of the ischemic infarct (Figure 1). However, distinct identification of the PIZ is difficult with routine histology, and few studies have been able to correlate the spatial relationship between altered biochemistry and distance from the ischemic infarct. ${ }^{7}$ Glial cell proliferation measured by GFAP immunohistochemistry is observed to surround the infarct, and can be used to approximate the location and size of the PIZ. ${ }^{11}$ Nonetheless, this is only one cellular marker and provides no 
information about the neurons in the PIZ. In addition to GFAP staining, H\&E histology revealed a staining pattern characteristic of edema (diffuse and patchy eosin staining) immediately adjacent to the infarct as has been observed by others using this model. ${ }^{5}$ Nevertheless, the true extent to which edema and altered tissue biochemistry extends from the infarct is difficult to gauge with histology alone. Traditionally, the gold standard in the field is to employ MRI to identify and study the PIZ. Previous MRI studies identified that the PIZ in this animal model is characterized with edema which extends up to a distance of $500 \mu \mathrm{m}$ away from the ischemic infarct. ${ }^{5}$ Even so, precise identification of the boundary between the PIZ and healthy tissue is extremely difficult given the low spatial resolution of MRI. Thus, while MRI is very well suited to studying in vivo longitudinal changes in the size and water content of both the PIZ and ischemic infarct, it reveals little information on the biochemical changes that correlate with these anatomical changes.

In this study the anatomical size and shape of the PIZ determined based on the distribution of total protein, total lipid, and ions such as $\mathrm{K}^{+}$and $\mathrm{Ca}^{++}$, paralleled the distribution identified previously by other studies using MRI. ${ }^{5}$ This is an important finding as it highlights that the scientific conclusions drawn about the size and anatomical alterations that occur within the PIZ should be translatable between MRI studies and those employing a spectroscopic imaging approach. The advantage of our spectroscopic imaging approach is that it reveals a wealth of biochemical information in addition to anatomic information regarding the size and shape of the PIZ. The main limitation being, that as with histology, spectroscopic imaging can only be performed ex vivo on tissue sections. In this study we have demonstrated how our spectroscopic imaging platform can be coupled with routine histology and immuno-

histochemistry to reveal a more complete anatomic and biochemical picture. Indeed, future studies would also benefit from incorporating MRI to provide accurate and robust in vivo imaging of the PIZ, alongside the alterations to neuron structure (histology) and cellular biochemical distribution (FTIR/XFI/Raman).

4.02 Elemental and molecular alterations within the PIZ are characteristic of edema and tissue swelling, but not oxidative stress

FTIR imaging and XFI revealed decreased total lipid, total protein and ions $\left(\mathrm{K}^{+}, \mathrm{Ca}^{++}\right)$ within the PIZ following ischemic insult. These results suggest decreased tissue density within 
this region and the loss of diffusible ions, consistent with tissue swelling in response to edema. Despite the significant decrease in lipid and protein content within the PIZ, the lipid to protein ratio was nearly identical within the PIZ relative to the contralateral hemisphere. The lack of a significant difference in the lipid to protein ratio is consistent with an overall decrease in tissue density caused by edema, rather than chemical alterations caused by oxidative lipid or protein damage or altered metabolism, which would be unlikely to preserve a constant lipid to protein ratio. This is in agreement with the histological findings which showed the characteristic eosin staining pattern induced by edema (i.e., diffuse and patchy eosin staining). Our results also support previous investigations by others that used MRI and histological evaluation of Evan's blue staining to reveal that vascular breakdown and edema are major physiological events within the infarct and PIZ in the first 24 hours following ischemic insult in this model. ${ }^{4,5,19,20}$ Although the imaging method used in this study cannot be used in in vivo, eliminating the possibility of longitudinal studies in the same animal, or the application to a clinical setting, a greater wealth of chemical information is provided relative to MRI. Specifically, the ability to quantitatively measure ion homeostasis within the PIZ using our spectroscopic imaging approach may have substantial use in future studies investigating the biochemical mechanisms of potential therapies. For example, as $\mathrm{Ca}^{2+}$ is a critical ion involved in neuro-transmission, future studies investigating therapeutic manipulation of the $\mathrm{Ca}^{2+}$ concentration within the PIZ could be performed with a combined MRI and spectroscopic imaging approach, but they could not be undertaken with MRI alone.

To further investigate the biochemical alterations within the PIZ, high resolution widefield SR-FTIR imaging was employed to image individual neurons. The results revealed that total protein is reduced within the neuron soma and neuropil within the PIZ, but the decreased lipid content is most pronounced within the neuropil and not the neuron soma. This is consistent with the known composition of neurons soma and neuropil. ${ }^{4,39,40,42}$ As the neuropil is relatively rich in lipids associated with membranes of the numerous dendrites as well as the myelin sheath of axons, swelling or loss of these structures in response to edema would produce a substantial decrease in lipid content. As the neuron soma is rich in protein, swelling of cell bodies in response to edema would be expected to lower the overall protein content per unit area. Raman spectroscopy imaging of the sub-cellular distribution of lipid acyl groups substantiated the widefield SR-FTIR imaging findings, confirming a significant decrease in lipid content within the 
neuropil. In addition, high resolution imaging of lipids using CARS revealed a reduction in the number of lipid rich myelinated axons in the tissue surrounding the infarct, providing further support for edema and tissue swelling. Axonal swelling associated with the early stages of Wallerian degeneration occurs within hours of the insult and likely contributes to the edema observed, whilst the subsequent loss of myelin and axons is significantly delayed in comparison and would not be observably at the time point examined here. ${ }^{66-68}$

It has been demonstrated in other studies that edema may correlate with oxidative stress and increased protein oxidation and protein misfolding, ${ }^{33}$ possibly driving cell degeneration. Consequently, the substantial amount of edema observed in the PIZ in this study could initiate protein misfolding and be a driving mechanism of ongoing neurodegeneration. However, the results from FTIR imaging revealed that although the relative aggregated protein content is increased within the ischemic infarct, there was not a significant increase in the aggregated protein content of the PIZ. This is an important finding as it suggests that oxidative stress potentially initiated by edema or other factors is not a driving mechanism of cell death within the PIZ at this time point. Similarly, although there was a net decrease in protein and lipid levels within the PIZ, there was no alteration in the lipid to protein ratio. This suggests these molecular alterations are not the result of chemical modification, which would likely alter the lipid to protein ratio. These results could not be obtained with traditional MRI alone as MRI is not sensitive to markers of oxidative stress. Biochemical assays could be used to determine that oxidative stress is occurring within the brain, but homogenization of the tissue removes all spatial information, and isolate via micro-dissection of the small PIZ region would be exceptionally difficult and inaccurate. Therefore, only a biospectroscopic imaging approach such as that used in this investigation is capable of revealing this level of biochemical detail. Due to the lack of chemical modification to lipids and proteins within the PIZ 24 hours after the ischemic insult, we speculate that the molecular and elemental alterations observed within the $\mathrm{PIZ}$ at this time point may be reversible, and therapeutic intervention aimed at minimizing edema may be beneficial. 


\subsection{Conclusions}

The results of this study show a distinct biomolecular and elemental gradient, which transitions between the ischemic infarct and healthy appearing tissue, which allows identification and delineation of the PIZ and characterization of the chemical alterations which occur within this zone. Critically, the size and appearance of the PIZ observed in this study correlates well previous MRI studies in the same model. Despite a signification decrease in the total lipid, total protein and elemental content within PIZ, increased levels of protein aggregates are not observed, in contrast to the ischemic infarct which shows a substantial increase in aggregated protein content. Together, these data indicate that oxidative-damage is not responsible for the observed molecular and elemental alterations within the PIZ. Rather, the molecular and elemental alterations are more consistent with tissue swelling following edema. This is further supported by the high resolution SR-FTIR imaging, Raman microscopy and CARS, which show decreased lipid content is most pronounced in the neuropil rather than the soma. This level of biochemical information could not be obtained with traditional methods. Therefore, the results of this study suggest that therapeutic interventions targeted at localized brain edema may be a successful strategy to mitigate tissue damage in the acute ischemic period, and that administration of anti-oxidant therapy may be of little benefit in rescuing the PIZ at this time point. Additionally, the results of this study suggest that the photothrombotic ischemic model may be highly valuable to study the biochemical mechanisms of edema following ischemic insult and to test the efficacy of novel treatments aimed at minimizing edema. Future studies, such as a time-course investigation are now required to investigate this avenue and to determine if this therapeutic strategy would translate well to the human condition.

\subsection{Acknowledgements}

This work was supported by a joint Canadian Institutes of Health Research (CIHR)/Heart and Stroke Foundation of Canada (HSFC) Synchrotron Medical Imaging Team Grant \#CIF 99472 awarded to H.N., M.E.K and others. M.J.H. is a CIHR Postdoctoral Fellow, a Saskatchewan Health Research Foundation postdoctoral fellow. M.J.H and S.C. are SMI postdoctoral fellows and CIHR-Training grant in Health Research Using Synchrotron Techniques (CIHR-THRUST) Fellows. Research described in this paper was performed in part at the Canadian Light Source, 
which is supported by the Natural Sciences and Engineering Research Council of Canada, the National Research Council Canada, the Canadian Institutes of Health Research, the Province of Saskatchewan, Western Economic Diversification Canada, and the University of Saskatchewan. Portions of this research were carried out at the Stanford Synchrotron Radiation Lightsource, a Directorate of SLAC National Accelerator Laboratory and an Office of Science User Facility operated for the U.S. Department of Energy Office of Science by Stanford University. The SSRL Structural Molecular Biology Program is supported by the DOE Office of Biological and Environmental Research, and by the National Institutes of Health, National Center for Research Resources, Biomedical Technology Program (P41RR001209). The Raman spectroscopic imaging for this research was performed at the Saskatchewan Structural Sciences Center (SSSC) at the University of Saskatchewan.

\subsection{Author Contributions}

*SC and MJH are equal first authors of this manuscript.

** denotes the co-author who acted as the primary supervisor for this research.

*SC contributed $20 \%$ of the experimental research, writing and editing the manuscript.

*MJH contributed $20 \%$ of experimental research, writing and editing the manuscript.

$\mathrm{HH}$ contributed $5 \%$ of the experimental research and contributed to editing the manuscript.

NJS contributed $5 \%$ of the experimental research and contributed to editing the manuscript.

BY contributed $5 \%$ of the experimental research and contributed to editing the manuscript.

AS contributed $5 \%$ of the experimental research and contributed to editing the manuscript.

ZI contributed $5 \%$ of the experimental research and contributed to editing the manuscript.

JM contributed $5 \%$ of the experimental research and contributed to editing the manuscript.

SK contributed $5 \%$ of the experimental research and contributed to editing the manuscript.

ZJ contributed $5 \%$ of the experimental research and contributed to editing the manuscript.

$\mathrm{HN}$ contributed $5 \%$ of the experimental research and contributed to editing the manuscript.

** MEK contributed $15 \%$ of the experimental research design and was the primary editor of the manuscript. 


\subsection{References}

1. Feigin, V.L. et al. Global and regional burden of stroke during 1990-2010: findings from the Global Burden of Disease Study 2010. Lancet 383, 245-54 (2014).

2. Lipton, P. Ischemic cell death in brain neurons. Physiol Rev 79, 1431-568 (1999).

3. Witte, O.W. \& Stoll, G. Delayed and remote effects of focal cortical infarctions: secondary damage and reactive plasticity. Adv Neurol 73, 207-27 (1997).

4. van Bruggen, $\mathrm{N}$. et al. T2- and diffusion-weighted magnetic resonance imaging of a focal ischemic lesion in rat brain. Stroke 23, 576-82 (1992).

5. Lee, V.M. et al. Evolution of photochemically induced focal cerebral ischemia in the rat. Magnetic resonance imaging and histology. Stroke 27, 2110-8; discussion 2118-9 (1996).

6. Carmichael, S.T. Rodent models of focal stroke: size, mechanism, and purpose. NeuroRx 2, 396409 (2005).

7. Katsman, D., Zheng, J., Spinelli, K. \& Carmichael, S.T. Tissue microenvironments within functional cortical subdivisions adjacent to focal stroke. J Cereb Blood Flow Metab 23, 997-1009 (2003).

8. Schroeter, M., Jander, S., Huitinga, I., Witte, O.W. \& Stoll, G. Phagocytic response in photochemically induced infarction of rat cerebral cortex. The role of resident microglia. Stroke 28, 382-6 (1997).

9. Jander, S., Schroeter, M. \& Stoll, G. Role of NMDA receptor signaling in the regulation of inflammatory gene expression after focal brain ischemia. J Neuroimmunol 109, 181-7 (2000).

10. Stoll, G., Jander, S. \& Schroeter, M. Inflammation and glial responses in ischemic brain lesions. Prog Neurobiol 56, 149-71 (1998).

11. Nowicka, D., Rogozinska, K., Aleksy, M., Witte, O.W. \& Skangiel-Kramska, J. Spatiotemporal dynamics of astroglial and microglial responses after photothrombotic stroke in the rat brain. Acta Neurobiol Exp (Wars) 68, 155-68 (2008).

12. Braun, J.S., Jander, S., Schroeter, M., Witte, O.W. \& Stoll, G. Spatiotemporal relationship of apoptotic cell death to lymphomonocytic infiltration in photochemically induced focal ischemia of the rat cerebral cortex. Acta Neuropathol 92, 255-63 (1996).

13. Carmichael, S.T. Cellular and molecular mechanisms of neural repair after stroke: making waves. Ann Neurol 59, 735-42 (2006).

14. Murphy, T.H. \& Corbett, D. Plasticity during stroke recovery: from synapse to behaviour. Nat Rev Neurosci 10, 861-72 (2009).

15. Kim, G.W., Sugawara, T. \& Chan, P.H. Involvement of oxidative stress and caspase-3 in cortical infarction after photothrombotic ischemia in mice. J Cereb Blood Flow Metab 20, 1690-701 (2000).

16. Nagayama, T. et al. Induction of oxidative DNA damage in the peri-infarct region after permanent focal cerebral ischemia. J Neurochem 75, 1716-28 (2000).

17. Hayashi, T., Sakurai, M., Itoyama, Y. \& Abe, K. Oxidative damage and breakage of DNA in rat brain after transient MCA occlusion. Brain Res 832, 159-63 (1999).

18. Provenzale, J.M., Jahan, R., Naidich, T.P. \& Fox, A.J. Assessment of the patient with hyperacute stroke: imaging and therapy. Radiology 229, 347-59 (2003).

19. Kuroiwa, T. et al. Development of a rat model of photothrombotic ischemia and infarction within the caudoputamen. Stroke 40, 248-53 (2009).

20. Pevsner, P.H. et al. A photothrombotic model of small early ischemic infarcts in the rat brain with histologic and MRI correlation. J Pharmacol Toxicol Methods 45, 227-33 (2001).

21. Dietrich, W.D., Ginsberg, M.D., Busto, R. \& Watson, B.D. Photochemically induced cortical infarction in the rat. 2 . Acute and subacute alterations in local glucose utilization. J Cereb Blood Flow Metab 6, 195-202 (1986). 
22. Leskovjan, A.C., Kretlow, A. \& Miller, L.M. Fourier transform infrared imaging showing reduced unsaturated lipid content in the hippocampus of a mouse model of Alzheimer's disease. Anal Chem 82, 2711-6 (2010).

23. Miller, L.M. et al. Synchrotron-based infrared and X-ray imaging shows focalized accumulation of $\mathrm{Cu}$ and $\mathrm{Zn}$ co-localized with beta-amyloid deposits in Alzheimer's disease. J Struct Biol 155, 30-7 (2006).

24. Kuzyk, A. et al. Association among amyloid plaque, lipid, and creatine in hippocampus of TgCRND8 mouse model for Alzheimer disease. J Biol Chem 285, 31202-7 (2010).

25. Liao, C.R. et al. Synchrotron FTIR reveals lipid around and within amyloid plaques in transgenic mice and Alzheimer's disease brain. Analyst 138, 3991-7 (2013).

26. Benseny-Cases, N., Klementieva, O., Cotte, M., Ferrer, I. \& Cladera, J. Microspectroscopy (muFTIR) reveals co-localization of lipid oxidation and amyloid plaques in human Alzheimer disease brains. Anal Chem 86, 12047-54 (2014).

27. Szczerbowska-Boruchowska, M. et al. Biomolecular investigation of human substantia nigra in Parkinson's disease by synchrotron radiation Fourier transform infrared microspectroscopy. Arch Biochem Biophys 459, 241-8 (2007).

28. Kastyak, M.Z. et al. Pigmented creatine deposits in Amyotrophic Lateral Sclerosis central nervous system tissues identified by synchrotron Fourier Transform Infrared microspectroscopy and Xray fluorescence spectromicroscopy. Neuroscience 166, 1119-28 (2010).

29. Heraud, P. et al. Early detection of the chemical changes occurring during the induction and prevention of autoimmune-mediated demyelination detected by FT-IR imaging. Neuroimage 49, 1180-9 (2010).

30. Hackett, M.J. et al. FTIR imaging of brain tissue reveals crystalline creatine deposits are an ex vivo marker of localized ischemia during murine cerebral malaria: general implications for disease neurochemistry. ACS Chem Neurosci 3, 1017-24 (2012).

31. Chwiej, J. et al. Synchrotron FTIR micro-spectroscopy study of the rat hippocampal formation after pilocarpine-evoked seizures. J Chem Neuroanat 40, 140-7 (2010).

32. Kutorasinska, J. et al. Differences in the hippocampal frequency of creatine inclusions between the acute and latent phases of pilocarpine model defined using synchrotron radiation-based FTIR microspectroscopy. Anal Bioanal Chem 405, 7337-45 (2013).

33. Hackett, M.J. et al. A new method to image heme-Fe, total $\mathrm{Fe}$, and aggregated protein levels after intracerebral hemorrhage. ACS Chem Neurosci 6, 761-70 (2015).

34. Gelfand, P., Smith, R.J., Stavitski, E., Borchelt, D.R. \& Miller, L.M. Characterization of Protein Structural Changes in Living Cells Using Time-Lapsed FTIR Imaging. Anal Chem 87, 6025-31 (2015).

35. Miller, L.M., Bourassa, M.W. \& Smith, R.J. FTIR spectroscopic imaging of protein aggregation in living cells. Biochim Biophys Acta 1828, 2339-46 (2013).

36. Hackett, M.J. et al. Novel bio-spectroscopic imaging reveals disturbed protein homeostasis and thiol redox with protein aggregation prior to hippocampal CA1 pyramidal neuron death induced by global brain ischemia in the rat. Free Radical Biology and Medicine 89, 806-818 (2015).

37. Barabas, M.E., Mattson, E.C., Aboualizadeh, E., Hirschmugl, C.J. \& Stucky, C.L. Chemical structure and morphology of dorsal root ganglion neurons from naive and inflamed mice. J Biol Chem 289, 34241-9 (2014).

38. Findlay, C.R. et al. Rapid biodiagnostic ex vivo imaging at 1 mum pixel resolution with thermal source FTIR FPA. Analyst 140, 2493-503 (2015).

39. Hackett, M.J. et al. A Sub-Cellular Biochemical Investigation of Purkinje Neurons Using Synchrotron Radiation Fourier Transform Infrared Spectroscopic Imaging with a Focal Plane Array Detector. ACS Chemical Neuroscience 4, 1071-1080 (2013). 
40. Kastyak-Ibrahim, M.Z. et al. Biochemical label-free tissue imaging with subcellular-resolution synchrotron FTIR with focal plane array detector. Neuroimage 60, 376-83 (2012).

41. Nasse, M.J. et al. High-resolution Fourier-transform infrared chemical imaging with multiple synchrotron beams. Nat Methods 8, 413-6 (2011).

42. Hackett, M.J., Caine, S., Liu, X., May, T.E. \& Borondics, F. Development of single-beam wide-field infrared imaging to study sub-cellular neuron biochemistry. Vibrational Spectroscopy 77(2015).

43. Amharref, $N$. et al. Discriminating healthy from tumor and necrosis tissue in rat brain tissue samples by Raman spectral imaging. Biochim Biophys Acta 1768, 2605-15 (2007).

44. Krafft, C., Neudert, L., Simat, T. \& Salzer, R. Near infrared Raman spectra of human brain lipids. Spectrochim Acta A Mol Biomol Spectrosc 61, 1529-35 (2005).

45. Lattermann, A. et al. Characterization of atherosclerotic plaque depositions by Raman and FTIR imaging. J Biophotonics 6, 110-21 (2013).

46. Matthaus, C. et al. In vivo characterization of atherosclerotic plaque depositions by Ramanprobe spectroscopy and in vitro coherent anti-stokes Raman scattering microscopic imaging on a rabbit model. Anal Chem 84, 7845-51 (2012).

47. Freudiger, C.W. et al. Multicolored stain-free histopathology with coherent Raman imaging. Lab Invest 92, 1492-502 (2012).

48. Leskovjan, A.C. et al. Increased brain iron coincides with early plaque formation in a mouse model of Alzheimer's disease. Neuroimage 55, 32-8 (2011).

49. Leskovjan, A.C., Lanzirotti, A. \& Miller, L.M. Amyloid plaques in PSAPP mice bind less metal than plaques in human Alzheimer's disease. Neuroimage 47, 1215-20 (2009).

50. Chwiej, J. et al. Investigations of differences in iron oxidation state inside single neurons from substantia nigra of Parkinson's disease and control patients using the micro-XANES technique. $J$ Biol Inorg Chem 12, 204-11 (2007).

51. Popescu, B.F. et al. Mapping metals in Parkinson's and normal brain using rapid-scanning x-ray fluorescence. Phys Med Biol 54, 651-63 (2009).

52. Lelie, H.L. et al. Copper and zinc metallation status of copper-zinc superoxide dismutase from amyotrophic lateral sclerosis transgenic mice. J Biol Chem 286, 2795-806 (2011).

53. Chwiej, J., Dulinska, J., Janeczko, K., Appel, K. \& Setkowicz, Z. Variations in elemental compositions of rat hippocampal formation between acute and latent phases of pilocarpineinduced epilepsy: an X-ray fluorescence microscopy study. J Biol Inorg Chem 17, 731-9 (2012).

54. Chwiej, J. et al. Progress of elemental anomalies of hippocampal formation in the pilocarpine model of temporal lobe epilepsy--an X-ray fluorescence microscopy study. Anal Bioanal Chem 404, 3071-80 (2012).

55. Chwiej, J. et al. The role of trace elements in the pathogenesis and progress of pilocarpineinduced epileptic seizures. J Biol Inorg Chem 13, 1267-74 (2008).

56. Chwiej, J. et al. X-ray fluorescence analysis of long-term changes in the levels and distributions of trace elements in the rat brain following mechanical injury. J Biol Inorg Chem 16, 275-83 (2011).

57. Carter, E.A. et al. Silicon nitride as a versatile growth substrate for microspectroscopic imaging and mapping of individual cells. Mol Biosyst 6, 1316-22 (2010).

58. Winship, I.R. \& Murphy, T.H. In vivo calcium imaging reveals functional rewiring of single somatosensory neurons after stroke. J Neurosci 28, 6592-606 (2008).

59. Hackett, M.J. et al. X-ray absorption spectroscopy at the sulfur K-edge: a new tool to investigate the biochemical mechanisms of neurodegeneration. ACS Chem Neurosci 3, 178-85 (2012).

60. Hackett, M.J. et al. Chemical alterations to murine brain tissue induced by formalin fixation: implications for biospectroscopic imaging and mapping studies of disease pathogenesis. Analyst 136, 2941-52 (2011). 
61. Hackett, M.J. et al. In situ biospectroscopic investigation of rapid ischemic and postmortem induced biochemical alterations in the rat brain. ACS Chem Neurosci 6, 226-38 (2015).

62. Webb, S.M. The MicroAnalysis Toolkit: X-ray Fluorescence Image Processing Software. in The 10th International Conference on X-ray Microscopy 196-199 (AIP Publishing., 2011).

63. Freudiger, C.W. et al. Label-free biomedical imaging with high sensitivity by stimulated Raman scattering microscopy. Science 322, 1857-61 (2008).

64. Evans, C.L. et al. Chemically-selective imaging of brain structures with CARS microscopy. Opt Express 15, 12076-87 (2007).

65. Pohling, C., Buckup, T., Pagenstecher, A. \& Motzkus, M. Chemoselective imaging of mouse brain tissue via multiplex CARS microscopy. Biomed Opt Express 2, 2110-6 (2011).

66. Beirowski, B., Nogradi, A., Babetto, E., Garcia-Alias, G. \& Coleman, M.P. Mechanisms of axonal spheroid formation in central nervous system Wallerian degeneration. $J$ Neuropathol Exp Neurol 69, 455-72 (2010).

67. Hinman, J.D. The back and forth of axonal injury and repair after stroke. Curr Opin Neurol 27, 615-23 (2014).

68. Thomalla, G. et al. Diffusion tensor imaging detects early Wallerian degeneration of the pyramidal tract after ischemic stroke. Neuroimage 22, 1767-74 (2004).

\section{Figures.}



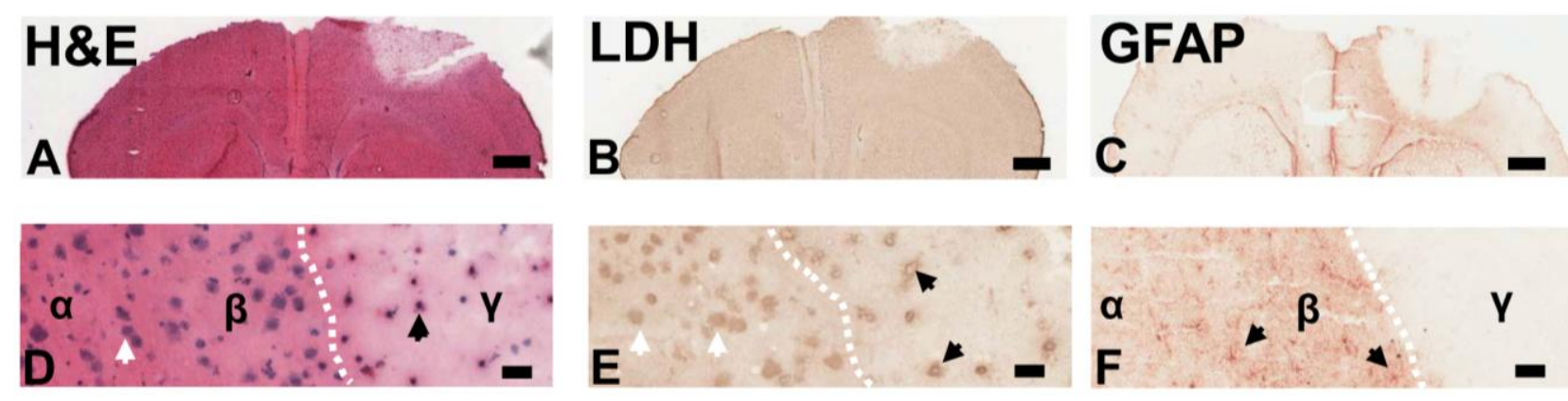

Figure 1: Routine histology and immunohistochemical evaluation of the ischemic infarct following photothrombotic stroke. (A-C) Overview of representative ipsilateral and contralateral hemispheres stained with (A) routine H\&E, (B) LDH immuno-staining, (C) GFAP immunostaining. (D-F) Higher magnification (20x) images of the infarct boundary shown in panels A-C. Neurons within the infarct in tissue sections stained with H\&E (black arrow, D) have a shrunken pkynotic nucleus, and display a bright pink eosinophilic cytoplasm, relative to healthy neurons (white arrow, D). LDH staining is absent from cells within the infarct with the exception of dark staining at the cell periphery (black arrows, E). Cells outside the infarct (white arrows, E) display normal morphology and stain evenly with LDH. A PIZ between the infarct and healthy appearing tissue can be observed in H\&E histology and GFAP immunohistochemical stained tissue sections. The neuropil is lightly stained with eosin within the infarct $(\gamma)$, and darkly stained with eosin in "healthy" appearing tissue $(\alpha)$, with intermediate staining observed in the PIZ $(\beta)$, characteristic of edema. No GFAP antigenicity is observed within the infarct $(\gamma)$, while mild antigenicity is observed in "healthy" appearing tissue $(\alpha)$, and intense GFAP antigenicity is observed in the PIZ $(\beta)$. Cells displaying GFAP antigenicity have typical glial cell morphology (black arrows, F). Scale bars $=500 \mu \mathrm{m}$ in A-C and $50 \mu \mathrm{m}$ in D-F. 

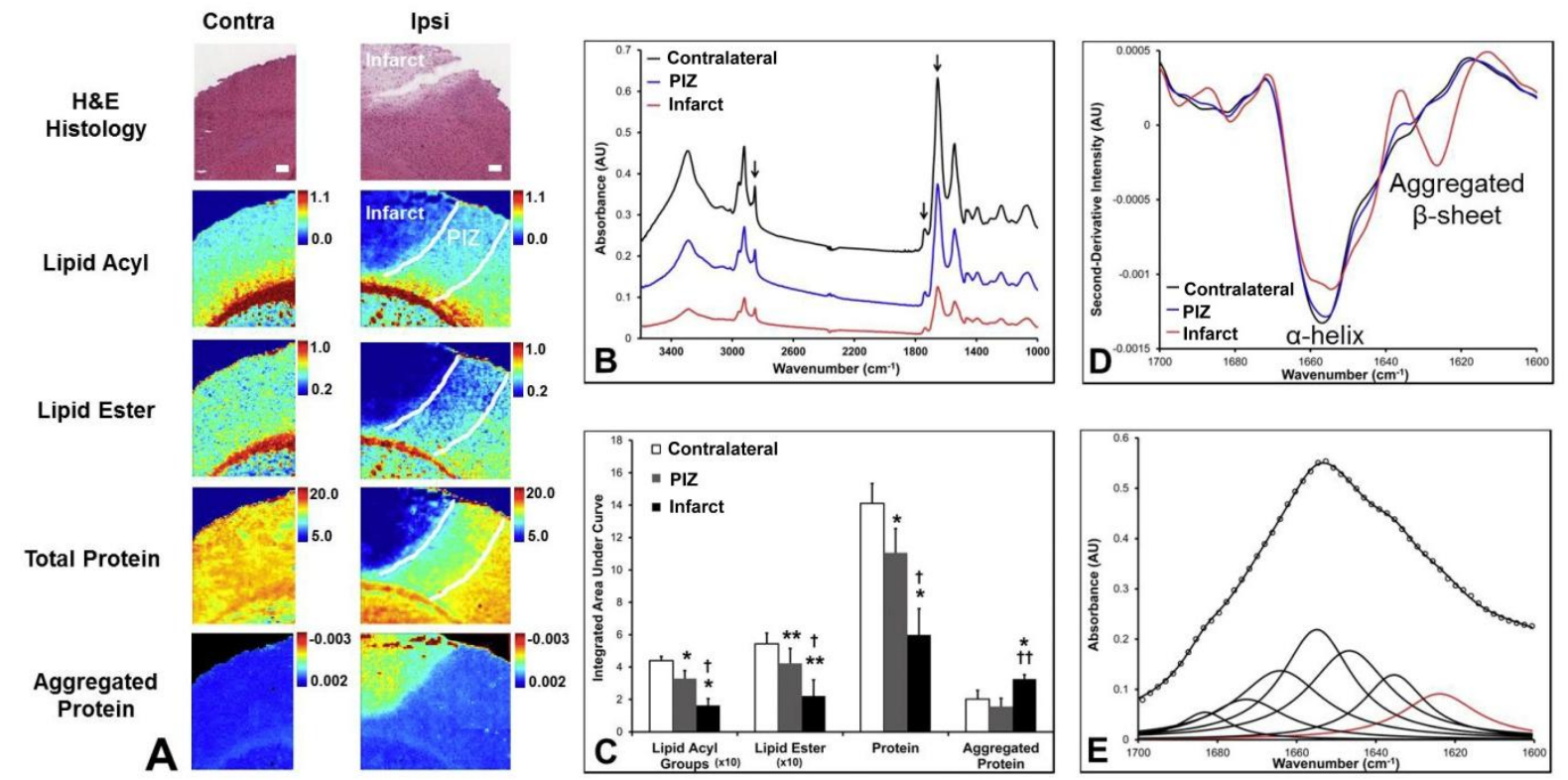

Figure 2: Globar-FTIR imaging of biochemical changes within contralateral and ipsilateral hemispheres following photothrombotic focal ischemic insult to the cortex. (A) Representative H\&E histology and globar FTIR images showing the distribution of lipid acyl groups, lipid esters and protein. Images of the lipid acyl, lipid ester and protein distribution were generated from the IAUC of the $v_{\mathrm{s}}\left(\mathrm{CH}_{2}\right)$ band $\left(2865-2845 \mathrm{~cm}^{-1}\right), v(\mathrm{C}=\mathrm{O})$ band $\left(1755-1715 \mathrm{~cm}^{-1}\right)$ and amide I band $\left(1700-1600 \mathrm{~cm}^{-1}\right)$, respectively. The location of the PIZ and infarct is highlighted by freehand drawn white lines. Scale bars $=100 \mu \mathrm{m}$. (B) Representative spectra averaged from the contralateral hemisphere, infarct and PIZ of one animal. Arrows (left to right) indicate peak maxima of the $v_{\mathrm{s}}\left(\mathrm{CH}_{2}\right)$ lipid acyl group absorption band $\left(2852 \mathrm{~cm}^{-1}\right)$, the $v(\mathrm{C}=\mathrm{O})$ lipid ester absorption band $\left(1738 \mathrm{~cm}^{-1}\right)$ and the amide I protein absorption band $\left(1656 \mathrm{~cm}^{-1}\right)$, respectively. (C) Representative second-derivative spectra of the amide I region, averaged from contralateral hemisphere, lesion and PIZ for one animal. (D) A representative example of the curve fitting process used to quantify the relative amount of aggregated protein. (E) Statistical analysis of the IAUC for lipid acyl group, lipid ester, and protein absorbance bands. The values for the lipid acyl group and the lipid esters have been multiplied by a factor of 10, to simply visual comparison with the total protein and aggregated protein levels. Statistical significance was determined from 6 animal replicates $(n=6)$ with a paired $t$-test and the $95 \%$ confidence interval. ${ }^{*} p<0.05,{ }^{*} p p<0.01$ relative to contralateral hemisphere. ${ }^{\dagger} p<0.05,{ }^{\dagger \dagger} p<0.01$ relative to PIZ. 

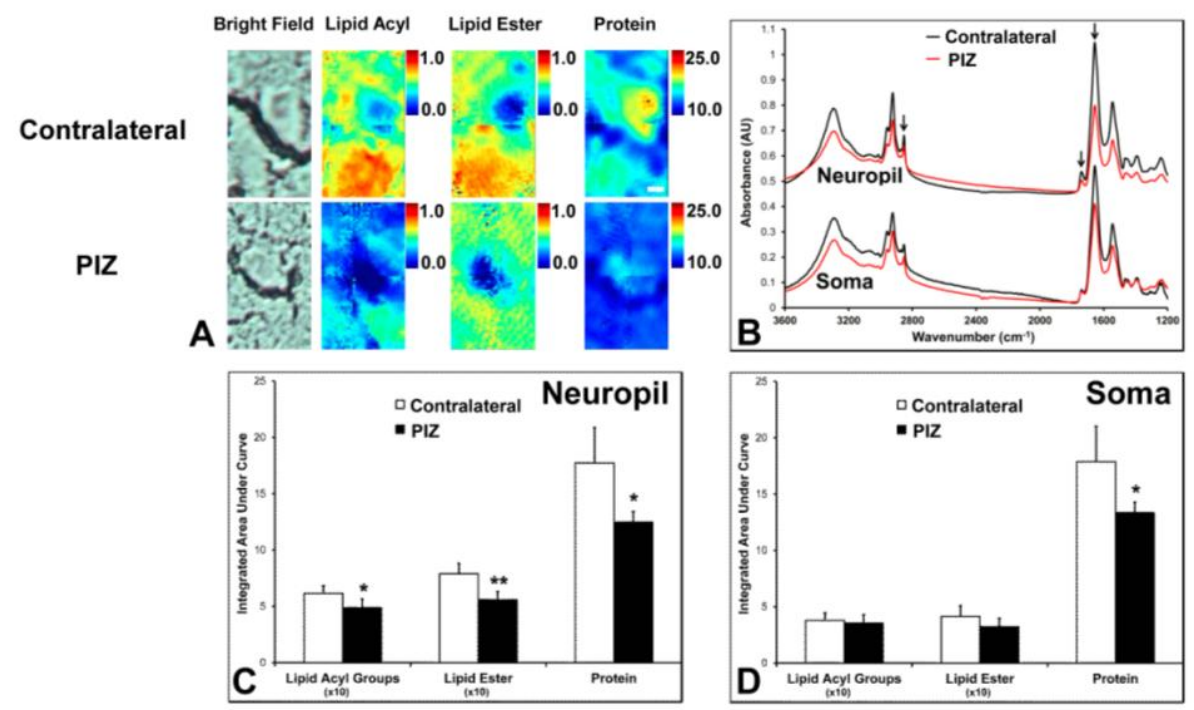

Figure 3: Wide-field SR-FTIR imaging of biochemical changes within individual neuron soma and the surrounding neuropil within the PIZ. (A) Representative bright field and SR-FTIR images showing the distribution of lipid acyl groups, lipid esters and protein for representative neurons located in the contralateral hemisphere and within the PIZ of the ipsilateral hemisphere. Images of the lipid acyl, lipid ester and protein distribution were generated from the IAUC of the $v_{\mathrm{s}}\left(\mathrm{CH}_{2}\right)$ band $\left(2865-2845 \mathrm{~cm}^{-1}\right), v(\mathrm{C}=\mathrm{O})$ band $\left(1755-1715 \mathrm{~cm}^{-1}\right)$ and amide $\mathrm{I}$ band $(1700-$ $1600 \mathrm{~cm}^{-1}$ ), respectively. See Supporting Information Figure 1 for anatomical location of regions of interest drawn to calculate the average spectra for neuron soma and surrounding neuropil. Scale bar $=5 \mu \mathrm{m}$. (B) Representative average spectra for a single neuron soma and surrounding neuropil in the contralateral hemisphere and within the PIZ of the ipsilateral hemisphere. Arrows (left to right) indicate location of the $v_{\mathrm{s}}\left(\mathrm{CH}_{2}\right)$ lipid acyl group absorption band $\left(2852 \mathrm{~cm}^{-1}\right)$, the $v(\mathrm{C}=\mathrm{O})$ lipid ester absorption band $\left(1738 \mathrm{~cm}^{-1}\right)$ and the amide I protein absorption band (1656 $\mathrm{cm}^{-1}$ ), respectively. (C and D) Quantification of the lipid acyl group, lipid ester, and protein absorbance bands. The values for the lipid acyl group and the lipid esters have been multiplied by a factor of 10, to simply visual comparison with the total protein and aggregated protein levels. Statistical significance was determined from 6 animal replicates $(n=6)$ with a paired $t$ test and the $95 \%$ confidence interval. $* p<0.05, * * p<0.01$. 


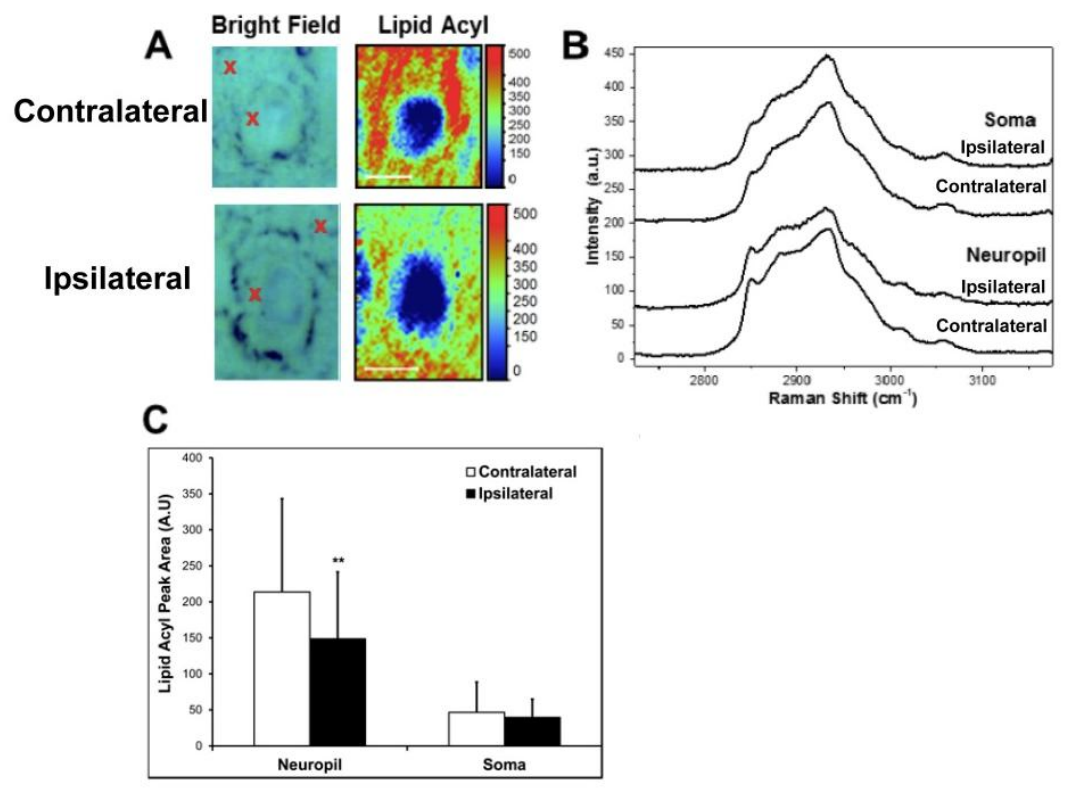

Figure 4: High resolution Raman images $(0.6 \times 0.6 \mu \mathrm{m}$ pixel size $)$ of lipid acyl within individual neuron soma and surrounding neuropil regions located in the contralateral hemisphere and the PIZ region of the ipsilateral hemisphere. (A) Representative bright field images of individual neurons located within the contralateral hemisphere and the PIZ region within the ipsilateral hemisphere. Raman images of lipid acyl distribution were generated by the $2830-2860 \mathrm{~cm}^{-1}$ peak area associated with the $v_{\mathrm{s}}\left(\mathrm{CH}_{2}\right)$ stretch. Scale bar $=10 \mu \mathrm{m}$. (B) Representative Raman spectra taken from neuropil and soma marked on the bright field images shown in (A). (C) Quantification of lipid acyl peak in soma and neuropil. Statistical significance was determined with a paired $t$-test and the $95 \%$ confidence interval. $* * p<0.01$. There is a large standard deviation in the absolute values of the lipid acyl peak area, and the major source of this variation was observed between tissue sections, hence a paired $t$-test was used for statistical analysis with neurons from the ipsilateral hemisphere paired with neurons from the contralateral hemisphere within the same tissue section. Approximately $80 \%$ of all neurons measured within the ipsilateral hemisphere displayed less lipid content than neurons in the contralateral hemisphere of the same tissue section. 

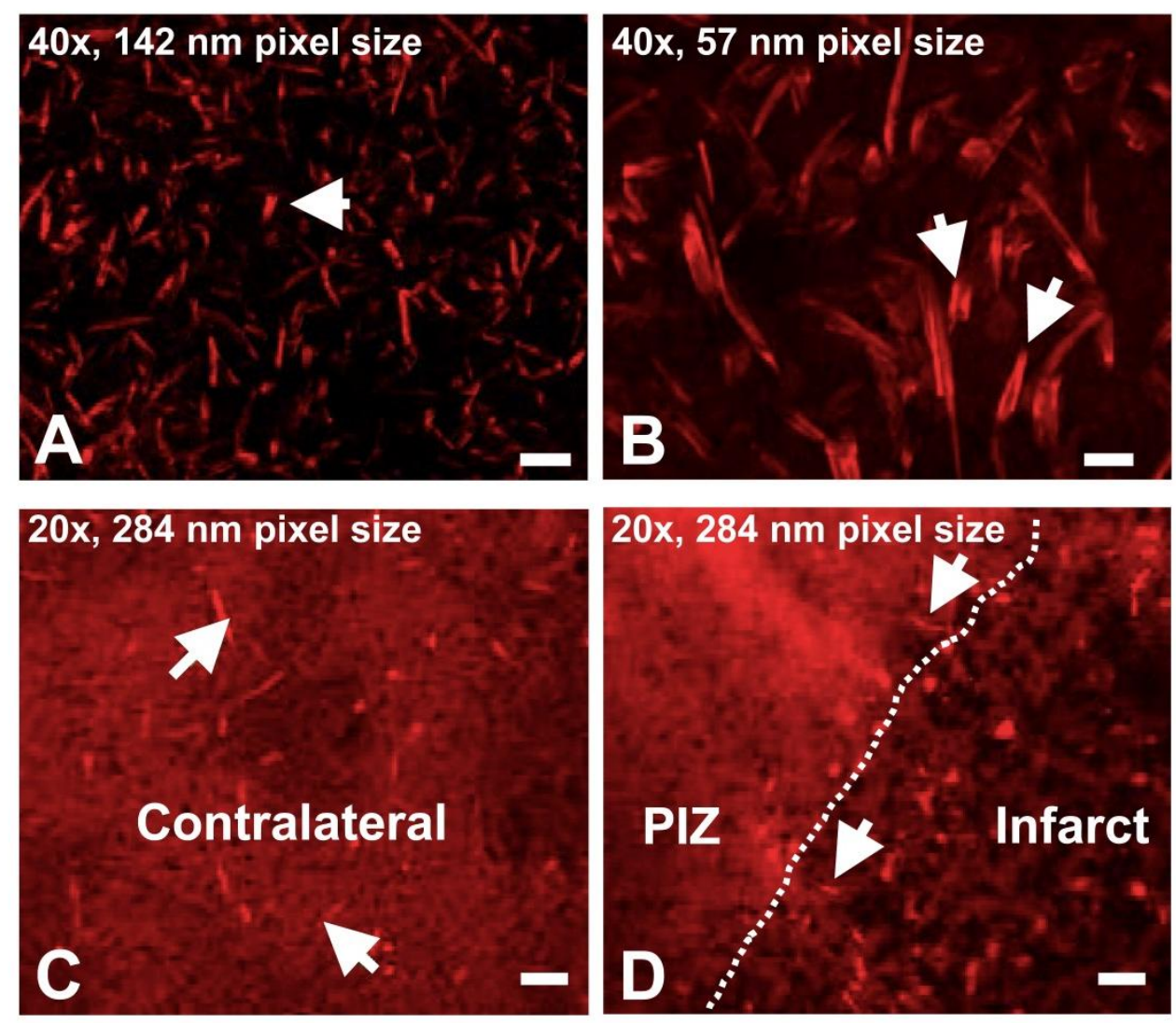

Figure 5: High resolution CARS imaging of lipids. (A-B) High resolution CARS imaging at 40× magnification with (A) $142 \mathrm{~nm}$ pixel size and (B) $57 \mathrm{~nm}$ pixel size, reveals characteristic distribution of lipids associated with the myelin sheath encasing axons (white arrows) in healthy tissue. (C) CARS imaging of the cortex in the contralateral hemisphere showing a relative abundance of myelinated axons compared to (D) the ipsilateral hemisphere where very few myelinated axons are observed inside the ischemic infarct or PIZ. Scale bar in A=10 $\mu \mathrm{m}, \mathrm{B}=5$ $\mu \mathrm{m}, \mathrm{C}, \mathrm{D}=25 \mu \mathrm{m}$. 

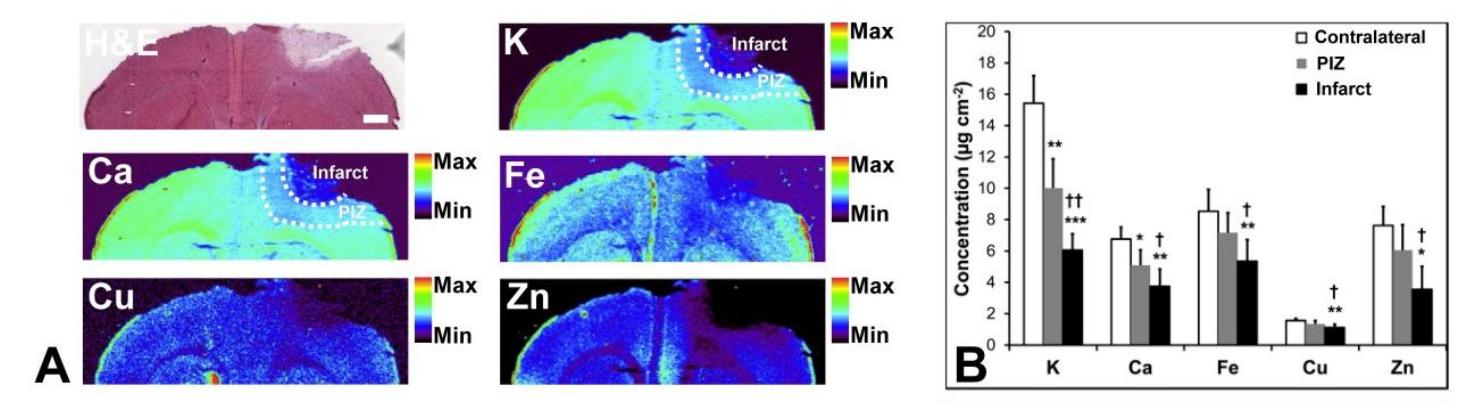

Figure 6: XFI elemental mapping following photothrombotic stroke. (A) Elemental maps of K, $\mathrm{Ca}, \mathrm{Fe}, \mathrm{Cu}$ and $\mathrm{Zn}$, within and around the ischemic infarct. Scale bar $=500 \mu \mathrm{m}$. (B) Quantification of the elemental changes in the ischemic infarct, PIZ and contralateral hemisphere. Statistical significance was determined from 6 animal replicates $(n=6)$ with a paired $t$-test and the $95 \%$ confidence interval. ${ }^{*} p<0.05$, $* * p<0.01$, *** $p<0.001$ relative to contralateral hemisphere. ${ }^{\dagger} p<0.05,{ }^{\dagger \dagger} p<0.01$ relative to PIZ. 